\title{
Broyden's Method for Solving Fuzzy Nonlinear Equations
}

\author{
Amirah Ramli, Mohd Lazim Abdullah, and Mustafa Mamat \\ Department of Mathematics, Faculty of Science and Technology, University of Malaysia Terengganu, \\ 21030 Kuala Terengganu, Malaysia
}

Correspondence should be addressed to Mustafa Mamat, mus@umt.edu.my

Received 8 February 2010; Revised 18 May 2010; Accepted 23 June 2010

Academic Editor: Ajith Abraham

Copyright ( 2010 Amirah Ramli et al. This is an open access article distributed under the Creative Commons Attribution License, which permits unrestricted use, distribution, and reproduction in any medium, provided the original work is properly cited.

Instead of using standard analytical techniques, like Buckley and Qu method, which are not suitable for solving a system of fuzzy nonlinear equations where the coefficient is fuzzy number, Broyden's method is proposed for solving fuzzy nonlinear equations. In this paper, an eight-step algorithm is used to solve fuzzy nonlinear equations. Two numerical examples are given to illustrate the proposed method.

\section{Introduction}

Over time, nonlinear equations have been played a major role in engineering, mathematics, medicines, and robotics. One of the applications in nonlinear problem is in weather which is inherently nonlinear in nature. Another interesting nonlinear problem is in phenomenon such as chaos arises from the difficulties of solving nonlinear equations. Consider the set of $n$ nonlinear equations

$$
f_{i}\left(x_{1}, x_{2}, \ldots, x_{n}\right)=0, \quad i=1,2, \ldots, n
$$

The general form of the nonlinear equation for $n=1$ may be simply stated as finding a value of the variable $x$ such that

$$
F(x)=0,
$$

where $F: \mathfrak{R}^{N} \rightarrow \mathfrak{R}^{N}$ and was denoted by $f_{i}$ is any nonlinear function of $x$. The value of $x$ is then called a solution or root of this equation and may be just one of many equations.

Numerical approximation methods are usually needed in solving system of equations when the equation is nonlinear. Solving a system of nonlinear equations is a problem that is avoided when possible, usually by approximating the nonlinear systems by a system of linear equations. But when this is unsatisfactory, the problem must be solved directly [1].

However, in most applications, the parameters of the system of nonlinear and measurement are not always represented by crisp number but also by fuzzy numbers.
Hence, it is necessary to study numerical method for solving fuzzy nonlinear equations. The concept of fuzzy number arises from the fact that many quantifiable phenomena do not lend themselves to being characterized in terms of absolutely precise numbers [2]. For example, standard analytical techniques proposed by [3] could not be suitable to solve the fuzzy nonlinear equations such as

(i) $a x^{4}+b x^{3}+c x^{2}-d x=e$,

(ii) $f x+g \cos x=h$,

(iii) $i x^{3}-j \sin x=k$,

where $a, b, c, d, e, f, g, h, i, j, k$, and $x$ are fuzzy numbers. Therefore, numerical methods for solving the above type equations are needed to be studied. In the study by [4-6], Newton's method and Steepest Descent's method were used to solve this type of equations.

The disadvantages of Newton's method arise from the need to calculate and invert the Jacobian matrix $J(x)$ at each iteration. Even Newton's method can converge very rapidly once a good initial value obtained, but it is not always easy to determine this kind of value and also this method is comparatively expensive to employ [1]. Although Steepest Descent's method does not require a good initial value its weakness is that it has low speed of convergence.

In this study, Broyden's method is proposed to solve this kind of equation. Broyden's method is superlinearly convergent. This method was chosen due to its being a powerful 
alternative to Newton's method, and hence it reduces the amount of calculation at each iteration without significantly degrading the speed of convergence. It replaces the Jacobian matrix $J$ with a matrix $A_{k-1}$ whose inverse is directly determined at each iteration, and it reduces the arithmetic operation form $O\left(n^{3}\right)$ to $O\left(n^{2}\right)$ [1]. Specifically the objective of this paper is to apply Broyden's method in solving fuzzy nonlinear equations.

Section 2 begins with some basic definition about fuzzy numbers and a brief overview of the fuzzy nonlinear equations. Section 3 describes definition of Broyden's method. Section 4 defines Broyden's method for solving nonlinear equations. Two numerical examples to illustrate the proposed method are given in Section 5. This paper ends with a short conclusion in Section 6.

\section{Preliminaries}

In this section, some important definitions of fuzzy numbers are reviewed.

2.1. Fuzzy Number. Fuzzy numbers are of great importance in fuzzy systems. The fuzzy numbers usually used in applications are the triangular (shaped) and the trapezoidal (shaped) fuzzy numbers [7].

Definition 1 (see $[8,9])$. A fuzzy number is set like $u: \mathfrak{R} \rightarrow$ $I=[0,1]$ which satisfies the following:

(1) $u$ is upper semicontinuous,

(2) $u(x)=0$ is outside some interval $[c, d]$,

(3) there are real numbers $a, b$ such that $c \leq a \leq b \leq d$ :

(3.1) $u(x)$ is monotonic increasing on $[c, a]$,

(3.2) $u(x)$ is monotonic decreasing on $[b, d]$,

(3.3) $u(x)=1, a \leq x \leq b$.

The set of all these fuzzy numbers is denoted by $E$. An equivalent parametric is also given in [10].

Definition 2 (see $[8,9])$. Fuzzy number $u$ in parametric form is a pair $(\underline{u}, \bar{u})$ of functions $\underline{u}(\alpha), \bar{u}(\alpha), 0 \leq \alpha \leq 1$, which satisfies the following requirements:

(1) $\underline{u}(\alpha)$ is a bounded monotonic increasing left continuous function,

(2) $\bar{u}(\alpha)$ is a bounded monotonic decreasing left continuous function,

(3) $\underline{u}(\alpha) \leq \bar{u}(\alpha), \quad 0 \leq \alpha \leq 1$.

Definition 3 (see $[8,9]$ ). Triangular fuzzy number $u=$ $(a, c, b)$ is a popular fuzzy number with the membership function

$$
u(x)= \begin{cases}\frac{(x-a)}{(c-a)}, & a \leq x \leq c, \\ \frac{(x-b)}{(c-b)}, & c \leq x \leq b,\end{cases}
$$

where $c \neq a, c \neq b$, and hence its parametric form is

$$
\underline{u}(\alpha)=a+(c-a) \alpha, \quad \bar{u}(\alpha)=b+(c-b) \alpha .
$$

Let $\operatorname{TF}(\Re)$ be the set of all trapezoidal fuzzy numbers. The addition and scalar multiplication of fuzzy numbers are defined by the extension principle and can be equivalently represented as follows [4-6].

For arbitrary $u=(\underline{u}, \bar{u}), v=(\underline{v}, \bar{v})$, and $k>0$, the addition $(u+v)$ and multiplication by real number $k>0$ are defined as

$$
\begin{array}{cc}
(\underline{u+v})(\alpha)=\underline{u}(\alpha)+\underline{v}(\alpha), \quad(\overline{u+v})(\alpha)=\bar{u}(\alpha)+\bar{v}(\alpha), \\
(\underline{k u})(\alpha)=k \underline{u}(\alpha), \quad(\overline{k u})(\alpha)=k \bar{u}(\alpha) .
\end{array}
$$

The Definitions 1, 2, and 3 are parameters of fuzzy nonlinear equations. In the next section, Broyden's method and its properties are presented.

\section{Broyden's Method}

Broyden's method is a method for solving the system of nonlinear equation and is designed to improve Newton's method with respect to storage and approximation of the Jacobian. Broyden's method is generalization of the secant method to multiple dimensions [1]. Once the initial value $x_{0}$ is obtained, then the next approximation $x_{1}$ is calculated as the same manner as Newton's method. In order to obtain $x_{2}$, the Secant method uses the finite difference approximation

$$
f^{\prime}\left(x_{n}\right) \approx \frac{f\left(x_{n}\right)-f\left(x_{n-1}\right)}{x_{n}-x_{n-1}}
$$

as a replacement for $f^{\prime}\left(x_{n}\right)$ in Newton's method by considering $x_{1}-x_{0}$ as a vector, and corresponding quotient is undefined. In Broyden's method, the Jacobian matrix $J\left(x_{1}\right)$ is replaced by matrix $A_{1}$ as follows:

$$
A_{1}\left(x_{1}-x_{0}\right)=F\left(x_{1}\right)-F\left(x_{0}\right) .
$$

Any nonzero vector in $\mathfrak{R}^{n}$ can be written as the sum of a multiple of $x_{1}-x_{0}$. How it acts on the orthogonal complement of $x_{1}-x_{0}$ is needed to be specified first in order to define the matrix $A_{1}$ as follows:

$$
A_{1} z=J\left(x_{0}\right) z, \quad \text { whenever }\left(x_{1}-x_{0}\right)^{t} z=0 .
$$

Thus, any vector orthogonal to $x_{1}-x_{0}$ is unaffected by the updated form $J\left(x_{0}\right)$, which was used to compute $x_{2} . A_{1}$ is defined by (7) and (8) as

$$
A_{1}=J\left(x_{0}\right)+\frac{\left[F\left(x_{1}\right)-F\left(x_{0}\right)-J\left(x_{0}\right)\left(x_{1}-x_{0}\right)\right]\left(x_{1}-x_{0}\right)^{t}}{\left\|x_{1}-x_{0}\right\|_{2}^{2}} .
$$

To determine $x_{2}$, the matrix $J\left(x_{1}\right)$ is replaced by $A_{1}$ as follows:

$$
x_{2}=x_{1}-A_{1}^{-1} F\left(x_{1}\right) \text {. }
$$


Once the $x_{i}$ is determined, then the new approximation $x_{i+1}$ is obtained by executing the following

$$
\begin{gathered}
A_{i}=A_{i-1}+\frac{y_{i}-A_{i-1} s_{i}}{\left\|s_{i}\right\|_{2}^{2}} s_{i}^{t}, \\
x_{i+1}=x_{i}-A_{i}^{-1} F\left(x_{i}\right),
\end{gathered}
$$

where $y_{i}=F\left(x_{i}\right)-F\left(x_{i-1}\right)$ and $s_{i}=x_{i}-x_{i-1}$. Hence, the number of scalar functional evaluations is reduced from $n^{2}+n$ to $n$ (those required for evaluating $F\left(x_{i}\right)$, but $O\left(n^{3}\right)$ calculations are still required to solve the associated $n \times n$ linear system:

$$
A_{i} s_{i+1}=-F\left(x_{i}\right) .
$$

The reduction form the quadratic convergence of Newton's method to superlinearly convergence can be improved by employing a matrix inversion formula of Sherman and Morrison [1].

Theorem 1 (see [1]. Sherman-Morrison). Let $A$ be a nonsingular matrix and let $x$ and $y$ be vectors, then $A+x y^{t}$ is nonsingular, provided that $y^{t} A^{-1} x \neq-1$, and

$$
\left(A+x y^{t}\right)^{-1}=A^{-1}-\frac{A^{-1} x y^{t} A^{-1}}{1+y^{t} A^{-1} x} .
$$

In order to eliminate the computation matrix inversion with each iteration, the Sherman-Morrison states that $A^{-1}$ can be computed directly from $A_{i-1}^{-1}$ by using this formula

$$
A_{i}^{-1}=A_{i-1}^{-1}+\frac{\left(s_{i}-A_{i-1}^{-1} y_{i}\right) s_{i}^{t} A_{i-1}^{-1}}{s_{i}^{t} A_{i-1}^{-1} y_{i}},
$$

where $s_{i}=x_{i}-x_{i-1}$ and $y_{i}=F\left(x_{i}\right)-F\left(x_{i-1}\right)$.

The following theorem defines that the Broyden' method make the smallest possible changes in updating the Jacobian.

Theorem 2 (see [11]). Among all matrices A satisfying $y_{i}=$ $A_{i+1} s_{i}$, the matrix $A_{n+1}$ minimizes the difference $\left\|A_{n+1}-A_{n}\right\|$.

Proof. By properties of the Euclidean norm and $\left\|s s^{t} / s^{t} \boldsymbol{s}\right\|=1$ for any vectors $s$, we have

$$
\begin{aligned}
\left\|A_{n+1}-A_{n}\right\| & =\left\|\frac{\left(y_{n}-A_{n} s_{n}\right) s_{n}^{t}}{s^{t} s}\right\| \\
& \leq\left\|A-A_{n}\right\|\left\|\frac{s_{n} s_{n}^{t}}{s_{n}^{t} s_{n}}\right\|=\left\|A-A_{n}\right\| .
\end{aligned}
$$

Therefore,

$$
A_{n+1} \in \arg \min \left\|A-A_{n}\right\|, \text { where } A: y_{k}=A s_{k} .
$$

Theorem 3 (see [12]). Suppose that the assumptions of Kantorovich's Theorem hold. Then there exist positive constants $\varepsilon$ and $\gamma$ s.t. if the starting point $x_{0}$ and the starting approximate Jacobian $A_{0}$ satisfy

$$
\left\|x_{0}-x^{*}\right\| \leq \gamma, \quad\left\|A_{0}-J\left(x^{*}\right)\right\| \leq \varepsilon,
$$

then the sequence generated by Broyden's method $\left\{x_{n}\right\}$ will converge superlinearly to $x^{*}$.
As stated previously, Broyden's method is a method for solving nonlinear equations. Next, tested Broyden's method is tested on solving fuzzy nonlinear equations.

\section{Broyden's Method for Solving Fuzzy Nonlinear Equations}

Now the objective is to obtain a solution for fuzzy nonlinear equations $F(x)=c$. The parametric form is given as follows.

$$
\begin{aligned}
& \underline{F}(\underline{x}, \bar{x} ; \alpha)=\underline{c}(\alpha), \quad \forall r \in[0,1] \\
& \bar{F}(\underline{x}, \bar{x} ; \alpha)=\bar{c}(\alpha) .
\end{aligned}
$$

To solve the nonlinear system $F(\underline{x}, \bar{x} ; \alpha)=0$, one initial approximation $x_{0}$ is given, and then generate a sequence $\left\{x_{n}\right\}$ which converges to the solution $x$, that is, $F(\underline{x}, \bar{x} ; \alpha)=0$. The method of Broyden's Newton can be described as follows:

Step 1. Transform the fuzzy nonlinear equations into parametric form.

Step 2. Solve the above system for $\alpha=0$ and $\alpha=1$ in order to obtain the initial value, $x_{0}$.

Step 3. Substitute $x_{0}$ into parametric form

Step 4. Compute the initial Jacobian matrix

$$
A_{0}(\alpha)=J\left(\underline{x}_{0}, \bar{x}_{0} ; \alpha\right) .
$$

Use it to compute the first approximation using Newton's method. Let $x_{k}(\alpha)=\left(\underline{x}_{k}, \bar{x}_{k} ; \alpha\right)$, then

$$
x_{1}(\alpha)=x_{0}(\alpha)-A_{0}(\alpha)^{-1} F\left(x_{0}(\alpha)\right) .
$$

For $k \geq 1$, suppose that $x_{1}(\alpha)$ has been obtained, then use the following steps to obtain $x_{2}(\alpha)$.

Step 5. Evaluate the function

$$
F\left(\underline{x}_{1}, \bar{x}_{1} ; \alpha\right)=\left[\begin{array}{l}
\underline{F}\left(\underline{x}_{1}, \bar{x}_{1} ; \alpha\right) \\
\bar{F}\left(\underline{x}_{1}, \bar{x}_{1} ; \alpha\right)
\end{array}\right] .
$$

Step 6. Update the approximate Jacobian using $s_{k}(\alpha)=$ $x_{k}(\alpha)-x_{k-1}(\alpha)$ and $y_{k}(\alpha)=F\left(x_{k}(\alpha)\right)-F\left(x_{k-1}(\alpha)\right)$, for $k=1,2, \ldots, n$.

Step 7. Use Sherman-Morrison formula to compute $A_{1}(\alpha)^{-1}$ as follows:

$A_{1}(\alpha)^{-1}=A_{0}(\alpha)^{-1}+\frac{\left[s_{1}(\alpha)-A_{0}(\alpha)^{-1} y_{1}(\alpha)\right] s_{1}(\alpha)^{t} A_{0}(\alpha)^{-1}}{s_{1}(\alpha)^{t} A_{0}(\alpha)^{-1} y_{1}(\alpha)}$.

Step 8. Solve the linear system $A_{1}(\alpha) x_{2}(\alpha)=x_{1}(\alpha)-F\left(x_{1}(\alpha)\right)$ for $x_{2}(\alpha)$.

Step 9. Repeat Steps from 4 to 8 and continue with the next $k$ until tolerance criteria $\varepsilon \leq 10^{-5}$ are satisfied. 


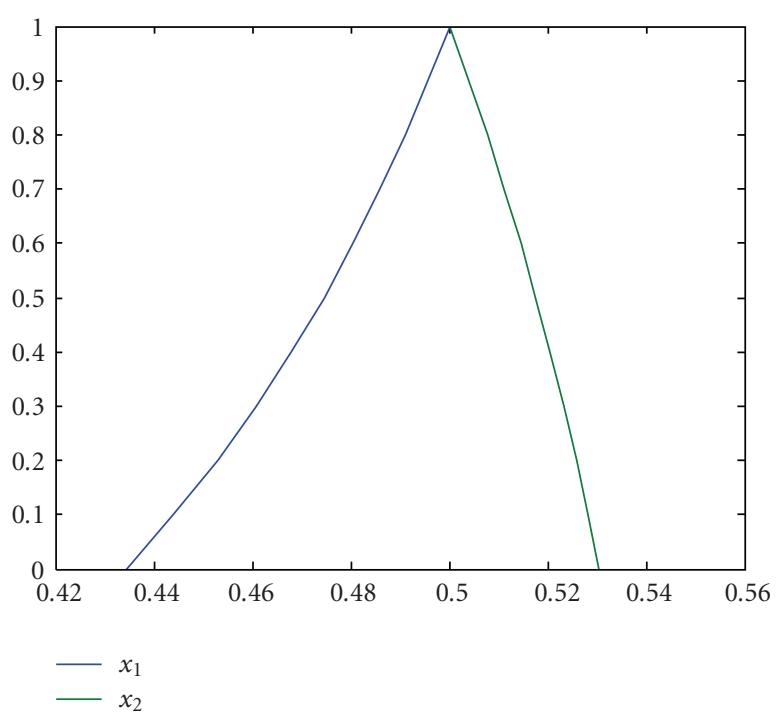

Figure 1: Solution of Broyden's Method for Example 1.

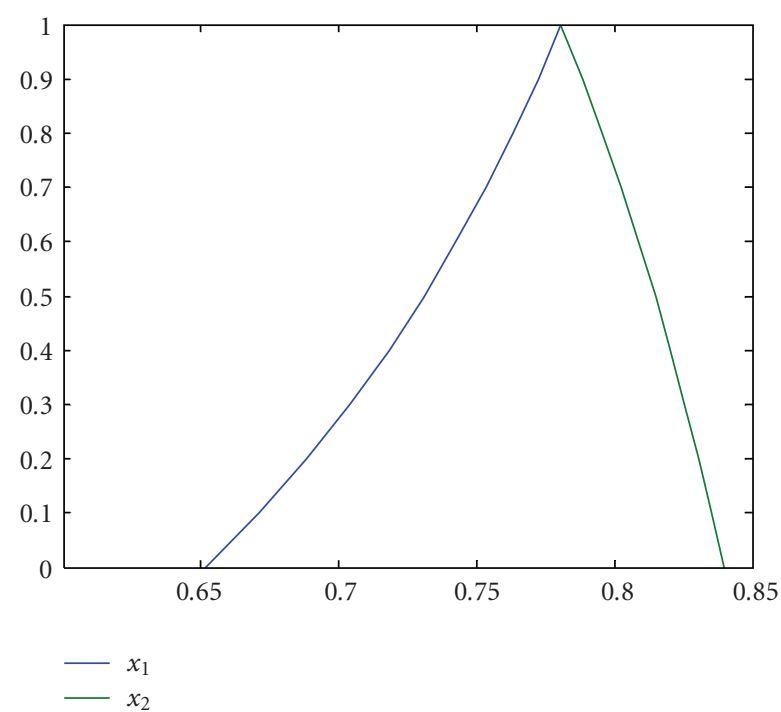

FIgURe 2: Solution of Broyden's Method for Example 2.

\section{Numerical Application}

Two examples are proposed to illustrate the Broyden's method for solving fuzzy nonlinear equations.

Consider the example from $[4,6]$.

Example 1. consider

$$
(3,4,5) x^{2}+(1,2,3) x=(1,2,3) .
$$

Assume that $x$ is positive, without any loss of generality, then the parametric form of this equation is as follows $[4,6]$ :

$$
\begin{aligned}
& (3+\alpha) \underline{x}^{2}(\alpha)+(1+\alpha) \underline{x}(\alpha)=(1+\alpha), \\
& (5-\alpha) \bar{x}^{2}(\alpha)+(3-\alpha) \bar{x}(\alpha)=(3-\alpha)
\end{aligned}
$$

or equality

$$
\begin{aligned}
& (3+\alpha) \underline{x}^{2}(\alpha)+(1+\alpha) \underline{x}(\alpha)-(1+\alpha)=0 \\
& (5-\alpha) \bar{x}^{2}(\alpha)+(3-\alpha) \bar{x}(\alpha)-(3-\alpha)=0 .
\end{aligned}
$$

The above system needs initial values as follows.For $\alpha=0$.

$$
\begin{aligned}
& 3 \underline{x}^{2}(0)+\underline{x}(0)=1, \\
& 5 \bar{x}^{2}(0)+3 \bar{x}(0)=3 .
\end{aligned}
$$

For $\alpha=1$ :

$$
\begin{aligned}
& 4 \underline{x}^{2}(1)+2 \underline{x}(1)=2, \\
& 4 \bar{x}^{2}(1)+2 \bar{x}(1)=2 .
\end{aligned}
$$

Hence, $\underline{x}(0)=0.4343, \bar{x}(0)=0.5307$, and $\underline{x}(1)=$ $\bar{x}(1)=0.5$. Suppose that $x$ is negative, where $\underline{x}(0)=$ $-0.7676, \bar{x}(0)=-1.1307$, and $\underline{x}(1)=\bar{x}(1)=-1$. For $\underline{x}(0)>\bar{x}(0)$, therefore, negative root does not exist.

For initial value, one can use the fuzzy number

$$
\begin{aligned}
x_{0} & =(\underline{x}(0), \underline{x}(1), \bar{x}(0)) \\
& =(0.4343,0.5,0.5307) .
\end{aligned}
$$

As can be observed, the above initial value is too close to the true solution. So this value can be taken as an actual solution. Therefore in this paper, another initial value is proposed in order to show the efficiency of the proposed method. Hence, the initial value is $x_{0}=(0.4,0.5,0.6)$. However, other initial values also can be used, but the suggested value is more reasonable to be used because Broyden's method needs a good initial value near to the exact solution that will lead to the true solution. Initially, parametric form can be written as follows:

$$
\begin{aligned}
& (3+\alpha) \underline{x}^{2}(\alpha)+(1+\alpha) \underline{x}(\alpha)-(1+\alpha)=0 \\
& (5-\alpha) \bar{x}^{2}(\alpha)+(3-\alpha) \bar{x}(\alpha)-(3-\alpha)=0 .
\end{aligned}
$$

Then substitute $x_{0}$ into parametric form. First iteration for Broyden's method is similar to the iteration for Newton's method. The Jacobian matrix for this fuzzy equation is

$$
\begin{gathered}
J\left(\underline{x}_{0}, \bar{x}_{0} ; \alpha\right) \\
=\left[\begin{array}{cc}
2(3+\alpha) \underline{x}(\alpha)+(1+\alpha) & 0 \\
0 & 2(5-\alpha) \bar{x}(\alpha)+(3-\alpha)
\end{array}\right] .
\end{gathered}
$$

Let $x_{0}=(0.4,0.5,0.6)$ and

$$
\begin{aligned}
& F\left(\underline{x}_{0}, \bar{x}_{0} ; \alpha\right) \\
& =\left\{\begin{array}{l}
\underline{F}\left(\underline{x}_{0}, \bar{x}_{0} ; \alpha\right), \\
\bar{F}\left(\underline{x}_{0}, \bar{x}_{0} ; \alpha\right)
\end{array}\right. \\
& =\left\{\begin{array}{l}
(3+\alpha) \underline{x}_{0}^{2}(\alpha)+(1+\alpha) \underline{x}_{0}(\alpha)-(1+\alpha)=0, \\
(5-\alpha) \bar{x}_{0}^{2}(\alpha)+(3-\alpha) \bar{x}_{0}(\alpha)-(3-\alpha)=0 .
\end{array}\right.
\end{aligned}
$$


Since $A_{0}(\alpha)=J\left(\underline{x}_{0}, \bar{x}_{0} ; \alpha\right)$

$$
A_{0}(\alpha)^{-1}=J\left(\underline{x}_{0}, \bar{x}_{0} ; \alpha\right)^{-1} .
$$

Let $x_{i}(\alpha)=\left(\underline{x}_{i}, \bar{x}_{i} ; \alpha\right)$, then

$$
x_{1}(\alpha)=x_{0}(\alpha)-A_{0}(\alpha)^{-1} F_{0}(\alpha)
$$

In the next step, $x_{1}(\alpha)$ is used to compute $x_{2}(\alpha)$. In Broyden's method, the Jacobian matrix is denoted by matrix $A_{i}^{-1}$, and it can be computed directly from $A_{i-1}^{-1}$ by using this formula

$A_{1}(\alpha)^{-1}=A_{0}(\alpha)^{-1}+\frac{\left[s_{1}(\alpha)-A_{0}(\alpha)^{-1} y_{1}(\alpha)\right] s_{1}(\alpha)^{t} A_{0}(\alpha)^{-1}}{s_{1}(\alpha)^{t} A_{0}(\alpha)^{-1} y_{1}(\alpha)}$,

where $s_{1}(\alpha)=x_{1}(\alpha)-x_{0}(\alpha)$ and $y_{1}(\alpha)=F\left(x_{0}(\alpha)\right)-$ $F\left(x_{0}(\alpha)\right)$ for $i=1,2, \ldots, n$. Repeat Steps from 4 to 8 until it satisfies the tolerance $\varepsilon \leq 10^{-5}$. After three iterations, the solution was obtained with maximum error $10^{-5}$. Details of the solution from $0 \leq \alpha \leq 1$ are given in Figure 1 .

Figure 1 shows that Broyden's method can converge very rapidly to a solution once initial value is obtained which is sufficiently close to true solution. The solution is obtained after two iterations with the maximum error less than $10^{-5}$.

Now the second example is proposed.

Example 2. consider

$$
(4,6,8) x^{2}+(2,3,4) x-(8,12,16)=(5,6,7) .
$$

Assume that $x$ is positive, without any loss of generality, then the parametric form of this equation is as follows $[4,6]$ :

$$
\begin{gathered}
(4+2 \alpha) \underline{x}^{2}(\alpha)+(2+\alpha) \underline{x}(\alpha)-(8+4 \alpha)=(5+\alpha), \\
(8-2 \alpha) \bar{x}^{2}(\alpha)+(4-\alpha) \bar{x}(\alpha)-(16-4 \alpha)=(7-\alpha),
\end{gathered}
$$

or equality

$$
\begin{aligned}
& (4+2 \alpha) \underline{x}^{2}(\alpha)+(2+\alpha) \underline{x}(\alpha)-(3+3 \alpha)=0 \\
& (8-2 \alpha) \bar{x}^{2}(\alpha)+(4-\alpha) \bar{x}(\alpha)-(9-3 \alpha)=0 .
\end{aligned}
$$

In order to obtain initial guess, we use above system. For $\alpha=$ 0 ,

$$
\begin{aligned}
& 4 \underline{x}^{2}(0)+2 \underline{x}(0)-3=0, \\
& 8 \bar{x}^{2}(0)+4 \bar{x}(0)-9=0 .
\end{aligned}
$$

For $\alpha=1$ :

$$
\begin{aligned}
& 6 \underline{x}^{2}(1)+3 \underline{x}(1)-6=0 \\
& 6 \bar{x}^{2}(1)+3 \bar{x}(1)-6=0 .
\end{aligned}
$$

Hence, $\underline{x}(0)=0.65139, \bar{x}(0)=0.83972$, and $\underline{x}(1)=\bar{x}(1)=$ 0.78078 . For initial guess, one can use the fuzzy number:

$$
\begin{aligned}
x_{0} & =(\underline{x}(0), \underline{x}(1), \bar{x}(0)) \\
& =(0.65139,0.78078,0.83972) .
\end{aligned}
$$

Consequently, the initial guess is $x_{0}=(0.65139,0.78078$, 0.83972).

Similar to the arguments in Example 1, the initial value above is too near to the true solution. Hence, other initial value is proposed as $x_{0}=(0.6,0.8,0.9)$, but other initial values may also be able to be tested as follows:

$$
\begin{aligned}
& (4+2 \alpha) \underline{x}^{2}(\alpha)+(2+\alpha) \underline{x}(\alpha)-(3+3 \alpha)=0, \\
& (8-2 \alpha) \bar{x}^{2}(\alpha)+(4-\alpha) \bar{x}(\alpha)-(9-3 \alpha)=0 .
\end{aligned}
$$

Then the above equations are solved by using Steps from 3 to 8 until the solution is obtained within the maximum error $\varepsilon \leq 10^{-5}$. Details of the solution for $0 \leq r \leq 1$ are shown in Figure 2 .

This figure illustrates how Broyden's method converges very rapidly to a solution, once initial value is obtained which is sufficiently close to true solution. However, it is not easy to determine initial values that will lead to a solution. Poor initial values may not lead to the actual solution. The solution using Broyden's method is obtained after three iterations with the maximum error less than $10^{-5}$.

\section{Conclusion}

In this paper, Broyden's method has been applied for solving fuzzy nonlinear equations. Firstly, fuzzy nonlinear equations are written in parametric form and then are solved using Broyden's method. The proposed eight steps algorithm yields the solution with maximum error less than $10^{-5}$. Two numerical examples have proven the feasibility of Broyden's method in solving fuzzy nonlinear equations.

\section{References}

[1] R. L. Burden and J. D. Faires, Numerical Analysis, Thomson, Pacific Grove, Calif, USA, 8th edition, 2005.

[2] G. J. Klir, U. St. Clair, and B. Yuan, Fuzzy Set Theory: Foundations and Applications, Prentice-Hall, Eaglewood Cliffs, NJ, USA, 1997.

[3] J. J. Buckley and Y. Qu, "Solving fuzzy equations: a new solution concept," Fuzzy Sets and Systems, vol. 39, no. 3, pp. 291-301, 1991.

[4] S. Abbasbandy and B. Asady, "Newton's method for solving fuzzy nonlinear equations," Applied Mathematics and Computation, vol. 159, no. 2, pp. 349-356, 2004.

[5] S. Abbasbandy and R. Ezzati, "Newton's method for solving a system of fuzzy nonlinear equations," Applied Mathematics and Computation, vol. 175, no. 2, pp. 1189-1199, 2006.

[6] S. Abbasbandy and A. Jafarian, "Steepest descent method for solving fuzzy nonlinear equations," Applied Mathematics and Computation, vol. 174, no. 1, pp. 669-675, 2006.

[7] J. J. Buckley, E. Eslami, and T. Feuring, Fuzzy Mathematics in Economics and Engineering, Physica, 2002.

[8] D. Dubois and H. Prade, Fuzzy Sets and Systems: Theory and Application, Academic Press, New York, NY, USA, 1980.

[9] H. J. Zimmermann, Fuzzy Set Theory and Its Application, Kluwer Academic Publishers, Dordrecht, The Netherlands, 1991.

[10] R. Goetschel Jr. and W. Voxman, "Elementary fuzzy calculus," Fuzzy Sets and Systems, vol. 18, no. 1, pp. 31-43, 1986. 
[11] J. E. Dennis and R. B. Schnabel, Numerical Methods for Unconstrained Optimization and Nonlinear Equations, Prentice-Hall, Eaglewood Cliffs, NJ, USA, 1983.

[12] S. J. Wright and J. Nocedal, Numerical Optimization, Springer, Berlin, Germany, 2nd edition, 2006. 

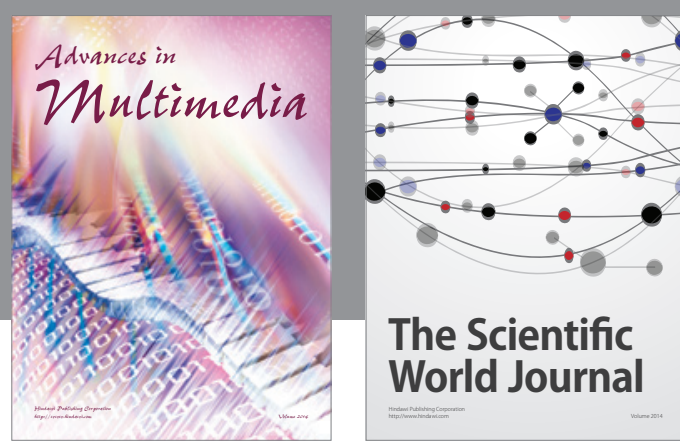

The Scientific World Journal
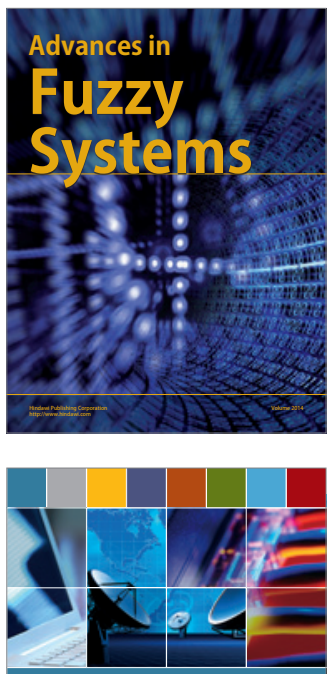

Computer Networks and Communications
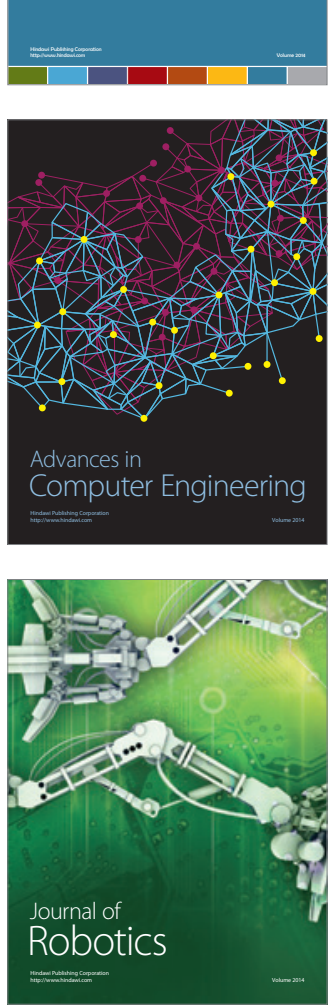
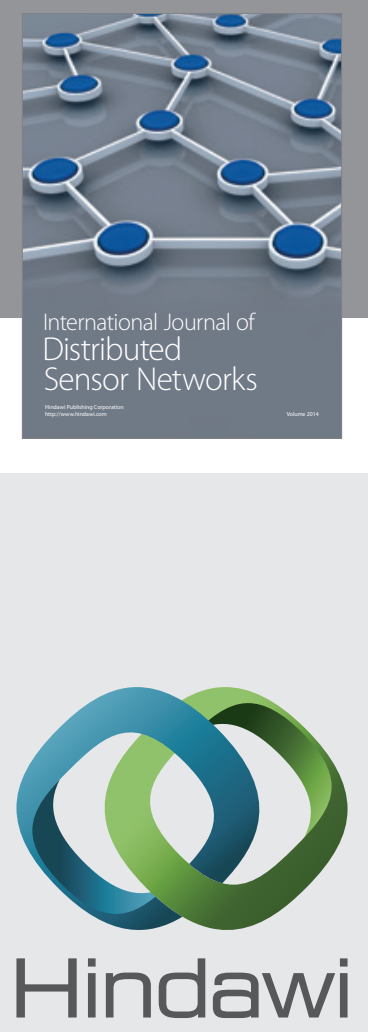

Submit your manuscripts at

http://www.hindawi.com
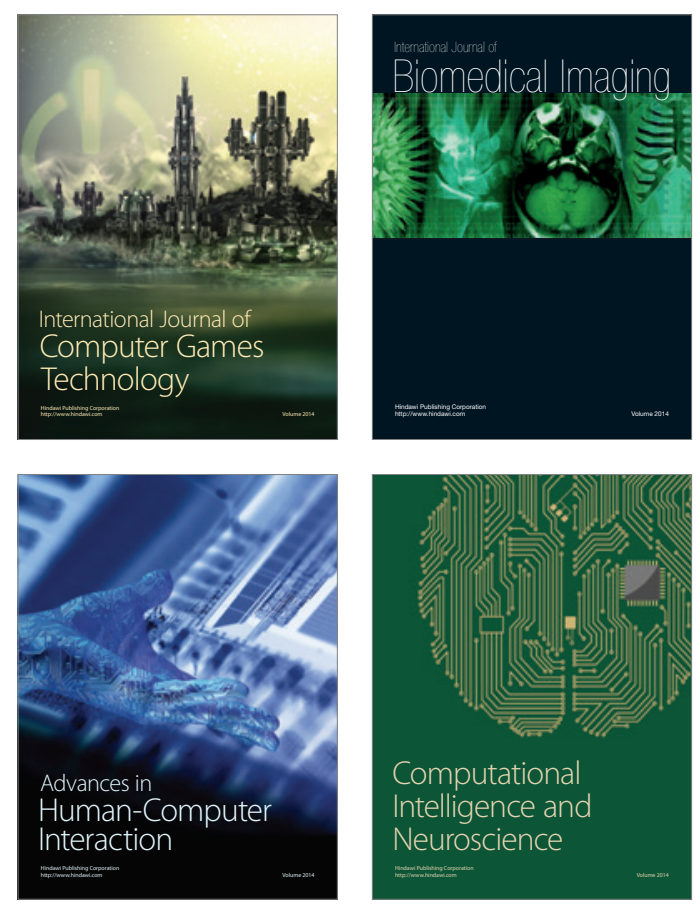
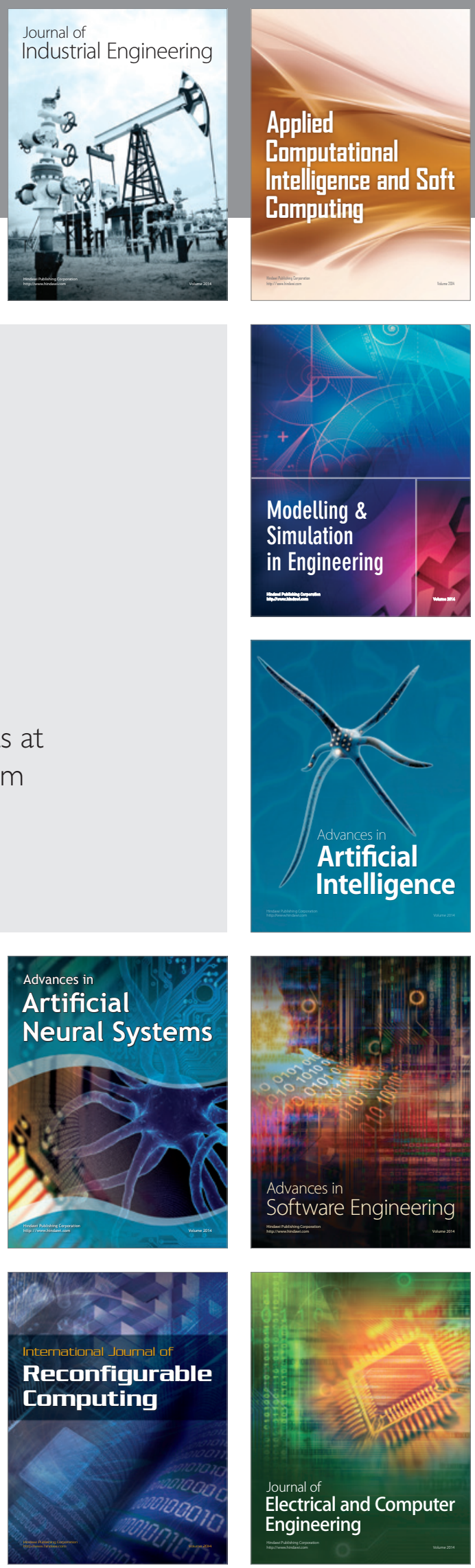